\title{
OBRAS PÚBLICAS REQUERIDAS VS. OBRAS PÚBLICAS EJECUTADAS: CASUÍSTICA DE LA REGIÓN CARIBE COLOMBIANA
}

\author{
REQUIRED PUBLIC WORKS VS. PUBLIC EXECUTED WORKS: \\ CASUISTRY OF THE COLOMBIAN CARIBBEAN REGION
}

\author{
María Camila Ayala Borrero ${ }^{1}$ \\ Yuliana Díaz Córdoba. ${ }^{2}$ \\ Diego Darío Pérez Ruíz ${ }^{3}$ \\ María Fernanda Serrano Guzmán 4
}

Fecha de recepción: 7 de mayo de 2017 - Fecha de aceptación: 5 de agosto de 2017

\begin{abstract}
Resumen
Las regiones naturales de Colombia son divisiones territoriales que se establecen en función de atributos geográficos como relieve, clima, vegetación, cultura, hidrografía, entre otros aspectos. En este artículo se presenta el resultado de la investigación adelantada acerca de la relación existente entre las necesidades detectadas en los planes de ordenamiento territorial (POT) y las obras de infraestructura ejecutadas en la región Caribe. Para ello, se revisaron los POTs de las ciudades capitales Riohacha, Cartagena, Barranquilla, Montería, Sincelejo, Santa Marta, Valledupar, Montería, y se compararon los proyectos formulados y ejecutados para dar respuesta a las problemáticas identificadas por las comunidades, información que debería estar disponible en la plataforma del Servicio Electrónico de Contratación (SECOP). Finalmente, el estudio reveló que las comunidades presentan necesidades insatisfechas en los sectores vial y de saneamiento básico y aun así los proyectos reportados y ejecutados corresponden a otros objetos, lo cual puede explicar la diferencia en el desarrollo de la región. Resulta cuestionable que, aunque las necesidades sean unas, los proyectos ejecutados sean otros, situación que repercute en el indicador de desempeño fiscal y de desarrollo municipal obtenido en estas comunidades. Palabras clave: Planes de ordenamiento territorial, Planificación del desarrollo, Gobierno local, Estrategias de desarrollo, Necesidades básicas.
\end{abstract}

\begin{abstract}
Colombia's natural regions are territorial divisions defined according to geographical attributes such as relief, climate, vegetation, culture, hydrography, among other aspects. This article shows the results of a research where was reviewed data regarding the relationship between needs identified in the territorial planning plans (TPP) and the infrastructure works carried out in the Caribbean region. For doing that, were reviewed the TPPs of the capital cities Riohacha, Cartagena, Barranquilla, Sincelejo, Santa Marta, Valledupar and Montería and it was checked if there was a relation between the projects, included in the EPSP Electronic Procurement Service platform, that were formulated and executed, effectively respond to the well-known problems identified by the communities. Finally, the study revealed that communities
\end{abstract}

\footnotetext{
1 Pontificia Universidad Javeriana Cali, mkmila.Ayala@hotmail.com

2 Pontificia Universidad Javeriana Cali, yuliana0475@hotmail.com

3 Pontificia Universidad Javeriana Cali, ddperez@javerianacali.edu.co 
continue with unsatisfied needs about road and basic sanitation sectors, and yet the projects reported and executed correspond to other objects, which may explain the difference in the development of the region. It is questionable that, although the needs are once, the projects executed are other, a situation that the indicator of fiscal performance and municipal development in these communities demonstrate. Keywords: National planning, Development planning, Local government, Development strategies, Basic needs.

\section{Introducción}

Colombia está organizada en regiones territoriales según diferentes atributos geomorfológicos, físicos, económicos, entre otros factores. La región Caribe colombiana (Figura 1) representa el 11.6\% del territorio nacional y está conformada por los Departamentos del Atlántico, Bolívar, Córdoba, Cesar, La Guajira, Magdalena, Sucre y San Andrés. Justamente, las ciudades capitales de estos departamentos tienen especial significado para el turismo tanto por los eventos culturales y musicales que se celebran como por los atractivos propios del entorno (Observatorio del Caribe Colombiano, 2015). Lamentablemente, factores como la falta de cobertura en servicios públicos y las condiciones inadecuadas de la infraestructura vial existente opacan las oportunidades para el desarrollo que se pueden lograr mediante el aprovechamiento de los paisajes que caracterizan esta región.

Figura 1

Región Caribe de Colombia

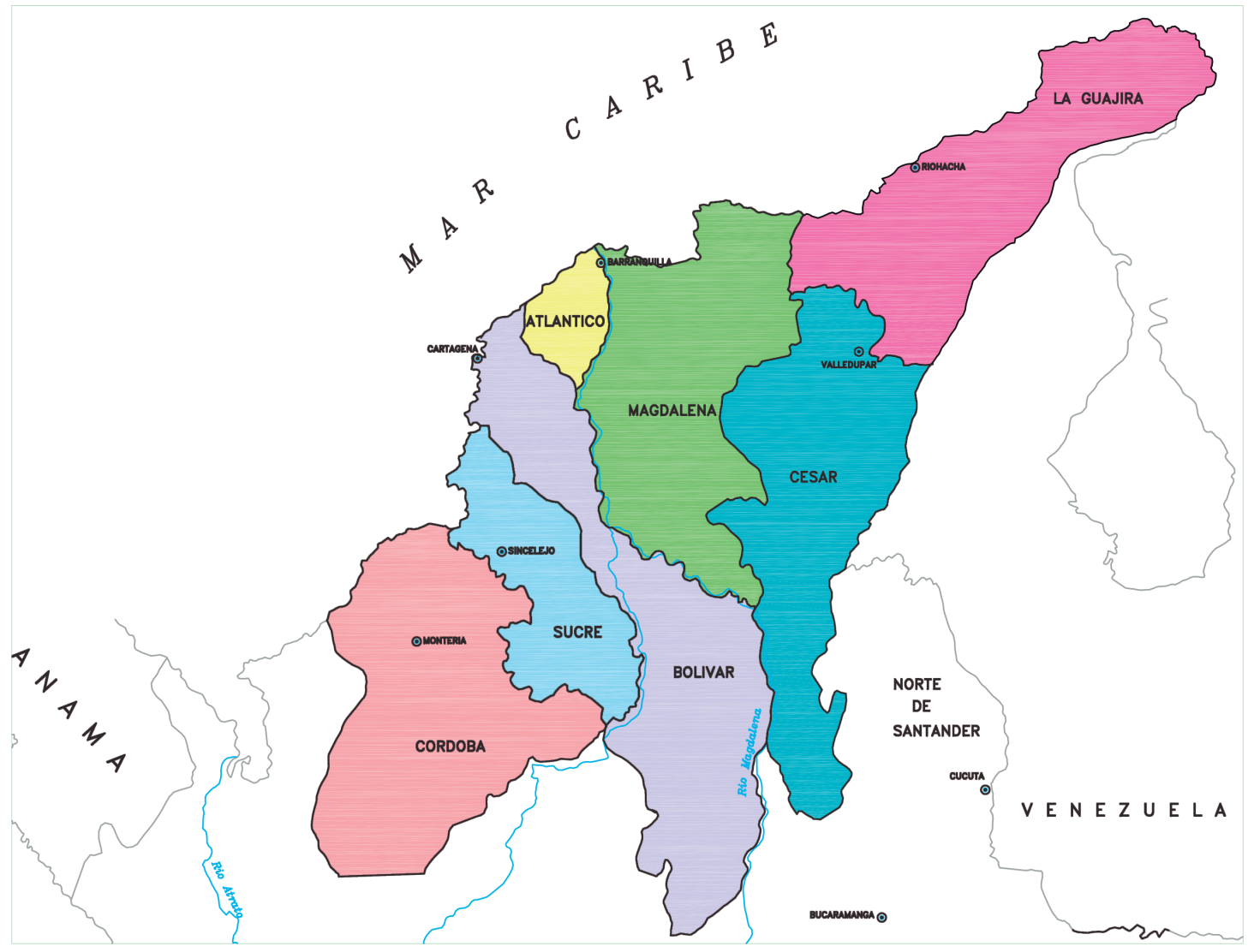

Fuente: elaboración propia 
En Colombia existe el Plan de Ordenamiento Territorial (POT) que es un instrumento técnico y normativo de planeación y gestión de largo plazo, el cual debe ser formulado por los municipios para la gestión de recursos de las distintas obras (Ministerio de Ambiente, Vivienda y Desarrollo Territorial, 2004). Justamente, el POT se elabora partiendo del diagnóstico de necesidades de las comunidades. Los proyectos contenidos en el POT surgen de un trabajo colaborativo entre diferentes actores encargados de la evaluación de los componentes: general, rural, urbano y de programación.

Dentro del componente general se incluyen atributos como sistemas de comunicación urbanorural, clasificación del suelo y definición de expansión, de áreas de reserva ambiental, zonas de riesgo. En el componente urbano se deben incluir las pautas para desarrollo físico de la ciudad con respecto a aspectos viales, de servicios públicos domiciliarios, de vivienda y normas urbanísticas. En cuanto al componente rural, se deben incorporar las políticas sobre utilización del suelo e interacción ruralcabecera municipal, incluyendo las áreas de conservación, de amenazas, de las zonas suburbanas y de los centros poblados rurales. También se debe considerar lo relacionado con servicios públicos y las normas para parcelación de predios. Finalmente, se definen las obras prioritarias, la programación de ejecución y los presupuestos correspondientes (Ministerio de Ambiente, Vivienda y Desarrollo Territorial, 2004).

Por otro lado, los informes de gestión de los municipios y la plataforma del sistema electrónico de contratación Pública (SECOP I) son instrumentos con los cuales el ciudadano puede realizar seguimiento a los proyectos que se han formulado para solucionar las problemáticas identificadas por las comunidades en las distintas vigencias, así como también corroborar la asignación de recursos que el ente gubernamental destinó para dichos proyectos.

Como se mencionó anteriormente, la información relacionada con los proyectos de las entidades estatales puede verificarse en el SECOP I, plataforma en donde dichas entidades deben publicar su actividad contractual. Lo anterior es de cumplimiento obligatorio por cuanto los contratos son ejecutados con recursos de los contribuyentes para producir bienes y servicios que beneficien a la ciudadanía, y en consecuencia todas las personas deben tener acceso a la información (Colombia compra eficiente, 2017). A pesar de los esfuerzos conjuntos comunidad-ente estatal, muchas de las necesidades detectadas son atendidas de manera parcial o, finalmente, son no programadas.

Este hecho motivó el desarrollo de esta investigación que tiene como objetivo central identificar la coincidencia entre los ejes temáticos declarados en los POTs de las ciudades capitales de la región Caribe colombiana y los proyectos que se han ejecutado y la repercusión de estas realidades en el desarrollo de la región.

\section{Metodología}

Para el desarrollo de esta investigación se revisaron los Planes de Ordenamiento Territorial de Barranquilla, Cartagena, Montería, Valledupar, Riohacha, Santa Marta, Sincelejo, San Andrés, ciudades capitales de los departamentos de Atlántico, Bolívar, Córdoba, Cesar, La Guajira, Magdalena, Sucre y San Andrés, respectivamente. Adicionalmente, se revisó en la plataforma del SECOP, las estadísticas relacionadas con los proyectos liquidados, para posteriormente revisar la relación con los proyectos ejecutados y las necesidades reportadas en los POTs.

\section{Hallazgos relevantes}

En este estudio se evaluaron los POTs de Riohacha, Sincelejo, Santa Martha, Valledupar, Cartagena, Barranquilla y Montería. En la Tabla 1 se resumen las problemáticas encontradas. 
Tabla 1

Problemática identificada y tipología de proyectos reportados en el SECOP

\begin{tabular}{lll}
\hline Ciudad & Problemática identificada & Tipo de proyectos encontrados en el SECOP*1 \\
\hline Riohacha & $\begin{array}{l}\text { Problemas ambientales, deficiencias en los sistemas } \\
\text { de saneamiento básico, baja calidad de las vías } \\
\text { secundarias. }\end{array}$ & $\begin{array}{l}\text { Construcción de pavimentos y andenes y cons- } \\
\text { trucción de un puente. }\end{array}$ \\
\hline Sincelejo & $\begin{array}{l}\text { Inconvenientes de movilidad, déficit de vivienda, } \\
\text { déficit en cobertura de servicios públicos y particu- } \\
\text { larmente de servicio de acueducto. }\end{array}$ & $\begin{array}{l}\text { Cuatro pavimentaciones, construcción de un } \\
\text { puente, construcción de un centro integral para } \\
\text { la comunidad, apoyo a un sector comercial. }\end{array}$ \\
\hline Santa Martha & $\begin{array}{l}\text { Repercusiones por inundaciones, déficit en cober- } \\
\text { tura de alcantarillado, desarrollo no planificado, } \\
\text { escasa disponibilidad de parqueaderos. }\end{array}$ & Tres mantenimientos viales. \\
\hline Valledupar & $\begin{array}{l}\text { Drenaje superficial ineficiente, colector de aguas } \\
\text { lluvias. }\end{array}$ & Mejoramiento vial. \\
\hline Cartagena & $\begin{array}{l}\text { Problemas sanitarios, restauración del paisaje, } \\
\text { expansibilidad. }\end{array}$ & $\begin{array}{l}\text { Estructura, mantenimiento de maquinaria, } \\
\text { mantenimiento vial. }\end{array}$ \\
\hline Barranquilla & $\begin{array}{l}\text { Accesibilidad, se requiere aumentar la cobertura de } \\
\text { los sistemas de saneamiento básico. }\end{array}$ & Construcción de un puente, mejoramiento vial. \\
\hline Montería & $\begin{array}{l}\text { Pérdida de recursos forestales, el municipio en } \\
\text { su zona rural se ve amenazado por inundaciones, } \\
\text { movimientos en masa y erosión. }\end{array}$ & Estudio, diseño y construcción de pavimentos. \\
\hline
\end{tabular}

Fuente: elaboración propia partiendo de información disponible en el SECOP

${ }^{*}$ Proyectos convocados bajo la modalidad de licitación Pública y que fueron liquidados entre 2014 y 2016

A continuación, se describen particularidades de cada ciudad y se especifican los proyectos que se ejecutaron:

\section{Riohacha (La Guajira)}

Riohacha es la capital del departamento de La Guajira y como "como el resto del país se halla en la zona intertropical o de latitudes bajas y está caracterizado por insignificantes variaciones térmicas a nivel mensual" (Departamento para la Prosperidad Social, Programa de las Naciones Unidas para el Desarrollo, Ministerio de Trabajo, Univesidad de la Guajira, 2013). Cuenta con zonas de resguardos indígenas, el Santuario de Flora y Fauna de los Flamencos y una parte que pertenece al Parque Nacional Natural Sierra de Santa Marta (Alcaldía de Riohacha, 2016).

En el POT se encuentran problemáticas como contaminación ocasionada por inadecuada disposición de los residuos sólidos en el litoral; problemas en las vías secundarias y terciarias; deficiencia en cuanto a servicios públicos, principalmente redes de acueductos y alcantarillado.

En el SECOP se encontraron dos proyectos ya liquidados, que son los siguientes:

- LP0132013 - Construcción de pavimentos y andenes; empalmes de alcantarillado pluvial y reparación de alcantarillado sanitario en diferentes barrios de Riohacha. (SECOP Proceso LP0132013, 2014)

- LP-SGT-SRN-027-2013 - Construcción del puente Río Cesar de la carretera Valledupar - Río Seco - San Juan de Cesar ruta 8004 a departamento de La Guajira. (SECOP Proceso LP-DOSRN-015-2014, 2016)

Estos proyectos responden parcialmente a las problemáticas que presenta el municipio en lo relacionado con infraestructura vial y adecuación de acueductos y alcantarillado, pero en la plataforma del SECOP no se evidencian proyectos para responder a las otras necesidades. 


\section{Sincelejo (Sucre)}

El municipio de Sincelejo, "capital cebuística de Colombia" (Ministerio de Agricultura y Desarrollo Rural República de Colombia, 2013) tiene un relieve irregular distribuido entre altitudes de 50 a 260 metros. Su calidad climática lo ubica como semi-seco y mega-térmico con vegetación natural y especies de palma de vino, samán, roble, cortadera, chaparro y macondo (Alcaldía de Sincelejo, 2016). La ciudad presenta problemas relacionados con la movilidad de tránsito, déficit de viviendas, déficit en prestación de servicios públicos. Se espera entonces, que se planteen proyectos de infraestructura que propendan por suplir estas necesidades. Al respecto, para el periodo de 2014 a 2015 se encontraron proyectos que buscaban mejorar la movilidad en los diferentes sectores de Sincelejo:

- LP-003-OP-2013 - Construcción del pavimento rígido, de la vía del corregimiento de San AntonioBuena Vistica- del K0+000 al K2+000, primera etapa en el municipio de Sincelejo, departamento de Sucre (SECOP Proceso LP-003-OP-2013, 2015).

- LP-005-OP-2014 - Construcción de obra de pavimento rígido y mejoramiento del entorno en el barrio Villa Mady, fase 1 municipio de Sincelejo Sucre (SECOP Proceso LP-005-OP-2014, 2015).

- LP-004-OP-2014 - Construcción de pavimento en concreto rígido en las calles 6 b entre carreras 14b y $14 \mathrm{c}$ - carrera $14 \mathrm{c}$ entre las calles $6 \mathrm{a}$ y $6 \mathrm{~b}$ carrera $14 \mathrm{c}$ entre las calles $6 \mathrm{~b}$ y $6 \mathrm{c}$ en el barrio san Luis de Sincelejo (SECOP Proceso LP-004-OP-2014, 2015).

- LP-010-2014 - Construcción del centro de integración ciudadana en el municipio Sincelejo - Sucre (SECOP Proceso LP-010-2014, 2015).

- LP-012-2014 - Construcción de pavimento en concreto rígido en el casco urbano del municipio del roble, departamento de Sucre (SECOP Proceso LP-012-2014, 2015).

- LP- SGT-OPA-052-2013 - Atención obras de emergencia para la construcción de los accesos del puente Pechelin en la carretera Sincelejo Toluviejo, en el departamento de Sucre. (SECOP Proceso LP-SGT-OPA-052-2013, 2014).

- LP-006-2013 - Apoyo producción y comercialización de cacao por parte de pequeños productores del departamento de Sucre (SECOP Proceso LP-006-2013, 2014).

Lamentablemente, en cuanto a la solución de déficit de viviendas y adecuación del sistema de alcantarillado para la mejoría de la distribución y calidad de servicios públicos no se encontraron proyectos reportados, es decir, los habitantes siguen presentando condiciones de vida no del todo favorables.

\section{Santa Marta (Magdalena)}

Santa Marta se cataloga como el puerto más profundo del continente americano, razón por la cual se beneficia por el transcurso y abordaje de barcos comerciales y turísticos. En esta zona se encuentra la Sierra Nevada y numerosos cuerpos de aguas que fluyen en las islas y bahías localizadas en el mar territorial (Alcaldía de Santa Marta, 2016). La belleza de sus paisajes se ve opacada por inundaciones, problemas de tipo sanitario, de accesibilidad y movilidad, de drenaje superficial urbano, de crecimiento no planificado y baja cobertura de servicios sanitarios, además de una sentida falta de espacio público y de áreas para parqueo de vehículos. Según el SECOP, estos son los proyectos que se han realizado para un mejoramiento de las problemáticas mencionadas:

- LP-DO-SRN-026-2014 - Mejoramiento y mantenimiento de la carretera vía alterna del puerto Santa Marta, sector Mamatoco, terminal marítimo, tramo (9007A), departamento de Magdalena (SECOP Proceso LP-DO-SRN-026-2014, 2016). 
- $\quad$ LP-DT-MAG-001-2014 - Mantenimiento rutinario a través de microempresas en las vías a cargo del instituto de vías, dirección territorial Magdalena (SECOP Proceso LP-DT-MAG-001-2014, 2016).

- $\quad$ LP-DT-ATL-001-2014 - Mantenimiento a través de microempresas en las vías a cargo del instituto de vías, dirección territorial Atlántico (SECOP LP-DT-ATL-001-2014, 2016).

Como se observa, los problemas atendidos estaban relacionados con la infraestructura vial, quedando pendiente las inversiones correspondientes al mejoramiento en cuanto a los servicios públicos, la potabilización del agua para los habitantes, entre otros aspectos.

\section{Valledupar (Cesar)}

Valledupar es centro para la producción agrícola, agroindustrial y ganadera en la región. Esta ciudad, reconocida por los eventos musicales, culturales y folclóricos de Colombia, simbólicamente, famosa como cuna del vallenato (Alcaldía de Valledupar, 2016), presenta problemas por transporte y disposición de aguas pluviales, lo cual da paso a las inundaciones que pueden afectar la salud y la calidad de vida de las personas; según el Plan de Ordenamiento Territorial de Valledupar, se hará la construcción del colector de la segunda etapa del plan maestro de acueducto y alcantarillado para habilitar las áreas de expansión en desarrollo concertado, es decir, adecuar los servicios públicos según el crecimiento poblacional.

Según el SECOP, estos son los proyectos que se han llevado a cabo en los últimos años:

- LP-DO-SRN-015-2014 - Mejoramiento y mantenimiento de carreteras Valledupar-Río Seco - San Juan del Cesar, sector pro + 000, ruta 8004 A en el departamento del Cesar. (SECOP Proceso LPDO-SRN-015-2014, 2015).

No se tienen datos concretos frente a la construcción del colector maestro de acueductos y alcantarillados ni se tienen datos de los avances, por lo cual se presume que no se ha concretado dicha construcción. Por otro lado, en la información revisada en diferentes documentos gubernamentales se encontraron reportes de ejecuciones de actividades de mantenimiento a la malla vial.

\section{Cartagena (Bolívar)}

Esta ciudad tiene especial interés turístico por la existencia de la muralla, el Castillo de San Felipe y posee un territorio insular conformado por las Islas de Tierra Bomba, Islas del Rosario, Barú, Islas de San Bernardo e Isla Fuerte (Alcaldía Distrital de Cartagena de Indias, 2016).

En este municipio se encuentran algunos problemas sanitarios, además de la restitución del paisaje de la zona urbana y problemas de expansibilidad. Los problemas mencionados anteriormente, se tomaron del POT, y han influido de manera negativa sobre la población que habita este territorio. A continuación, se muestran los proyectos que fueron consultados en el SECOP:

- UNIBAC-LIC-002-2016 - Consolidación de la cubierta en el proceso de restauración monumental del convento de San Diego, declarado bien de interés cultural de carácter nacional (SECOP Proceso UNIBAC-LIC-002-2016, 2016).

- LIC-SI-012-2014 - Administración y operación de la maquinaria pesada y volquetas a cargo del departamento de Bolívar para la implementación del banco de maquinaria destinado a la atención de emergencias, puntos críticos y mantenimientos preventivos de la red vial en el departamento de Bolívar (SECOP Proceso LIC-SI-012-2014, 2015). 
- LP-DT-ATL-001-2014 - Mantenimiento rutinario a través de microempresas en las vías a cargo del Instituto Nacional de las Vías Dirección Territorial Atlántico (SECOP Proceso LP-DTATL-001-2014, 2016).

Es importante resaltar que los procesos que aparecen liquidados en el SECOP no resuelven completamente las problemáticas mencionadas en el POT. Existen problemas que están afectando la calidad de vida de los habitantes, además que repercuten en el ejercicio de la actividad turística.

\section{Barranquilla (Atlántico)}

Esta ciudad está bañada por el mar Caribe y por la margen izquierda del río Magdalena en su trayecto final. Se caracteriza por un clima tropical de tipo estepa y árido (Gobernación de Atlántico, 2016), cuyas "principales ventajas son el clima y el paisaje, que han demostrado ser algunos de los principales factores determinantes del crecimiento de las ciudades" (Banco de la República, Gersón Javier Pérez y otros., 2014).

Este municipio también cuenta con problemas de accesibilidad vial y peatonal. En el POT se plantean programas para dar solución a las siguientes problemáticas: agua potable, acueducto y alcantarillado, alcantarillado pluvial, recolección transporte de residuos sólidos.

Según el SECOP existen dos contratos liquidados bajo la modalidad de licitación pública:

- LP-DO-SRN-024-2014 - Mejoramiento y mantenimiento de los accesos y orejas al puente Laureano Gómez (ruta 9007), accesos y orejas al puente Olaya herrera (ruta 90a-01) y accesos y orejas al puente de la cordialidad (ruta 9006) departamento de Atlántico (SECOP Proceso LP-DOSRN-024-2014, 2016).

- LP-DT-ATL-001-2014 - Mantenimiento rutinario a través de microempresas en las vías a cargo del instituto nacional de las vías dirección territorial Atlántico (SECOP Proceso LP-DT-ATL-001-2014, 2016).

Como se puede observar en los proyectos realizados según el SECOP, no se encuentra inversión en el tema de acueductos y alcantarillados; se han priorizado las soluciones a los problemas viales.

\section{Montería (Córdoba)}

Esta ciudad es activamente ganadera (Alcaldía de Montería, 2015) y actualmente cuenta con la ronda del Sinú, un espacio construido para "embellecer y a aumentar los espacios para la recreación" (Banco de la República, Gersón Javier Pérez y otros., 2014). Dentro de los problemas identificados está la pérdida de recursos forestales y las inundaciones que amenazan las zonas rurales. Además, problemas asociados a movimientos en masa y erosión.

Según el SECOP, estos son los proyectos que se han realizado en los últimos años en la ciudad de Montería:

- LP-042-2015 - Estudio, diseño y construcción de pavimento en las siguientes calles: 1) Calle 19 entre carreras $6-8^{\mathrm{a}}$ Barrio Colón 2) Calle 18 entre carrera 6-8 ${ }^{\mathrm{a}}$ Barrio Colón 3) Diagonal 4 entre transversal 1-3 4) Transversal 3 entre diagonal 4-5 Barrio Policarpa y reposición de losas de la calle 13 con carrera 13 en la ciudad de Montería, departamento de Córdoba (SECOP Proceso L.P. 0422015, 2015).

Aunque en el POT no se mencionan problemas de infraestructura vial, el POT del municipio de Montería propone cinco proyectos de infraestructura vial y transporte, servicios de recreación, 
abastecimiento, alimentos y funciones industriales (Alcaldía de Montería, 2009). Con respecto al tema vial y según el SECOP, parece ser que los proyectos propuestos se han ejecutado a cabalidad. Sin embargo, en cuanto al tema medioambiental de deforestación e inundación no se ha tomado ninguna medida, situación preocupante para los habitantes.

\section{Discusión}

Las comunidades realizan un ejercicio diagnóstico de necesidades y usualmente reportan lo encontrado en los planes de desarrollo. Para el mejoramiento del país, lo ideal sería que se formularan y ejecutaran proyectos tendientes a la búsqueda de soluciones a las problemáticas identificadas. Se debe destacar aquí que, si bien es cierto que los mantenimientos viales son necesarios para una mayor conectividad en las regiones y una mejor comercialización de los productos, además de los proyectos ejecutados que apuntan a mejorar las condiciones viales deben plantearse programas que solucionen las problemáticas de saneamiento básico, de déficit de vivienda y de mejoramiento a la calidad de vida en general de las personas. Además, según un estudio adelantado por el Banco de la República, la tendencia es que en las comunidades se refuerce el equipamiento deportivo, recreativo y cultural ("amenities") que inciden favorablemente en la calidad de vida (Banco de la República, Gersón Javier Pérez y otros., 2014), por lo cual este tipo de proyectos también deberían planearse.

Ahora bien, en la plataforma del SECOP no se encuentran en su totalidad los proyectos ejecutados según las necesidades identificadas en el POT, pero es posible identificar indicadores de desarrollo y crecimiento por otros medios, lo cual, aparentemente, deja entrever que las obras se están ejecutando. Uno de estos indicadores es de desempeño integral municipal (IDIM) del cual, por ejemplo, el Departamento Nacional de Planeación realiza anualmente un informe, esto con el fin de evaluar la gestión pública, la toma de decisiones de política pública y de asignaciones de recursos según la problemática local identificada por cada departamento y por ende de cada municipio (Departamento Nacional de Planeación Grupo de Estudios Territoriales, 2016).

Este indicador considera eficacia, eficiencia, cumplimiento de requisitos legales y gestión, en virtud de lo cual se establecen las siguientes ponderaciones en función del nivel de cumplimiento: sobresaliente (IDIM $\geq 80 \%$ ), satisfactorio (IDIM $\geq 70$ y menor a $80 \%$ ), medio (IDIM $\geq 60$ y menor a $70 \%$ ), bajo (IDIM $\geq 40$ y menor a $60 \%$ ) y crítico (IDIM menor del $40 \%$ ). El promedio nacional fue de $71.4 \%$, es decir satisfactorio. Si se compara el promedio nacional con el regional se observa que solamente el Departamento del Atlántico demostró un IDIM satisfactorio (Tabla 2).

Tabla 2

Indicador de Desempeño Integral Municipal-Promedio Municipal por Departamentos

\begin{tabular}{cc}
\hline Departamento & $\begin{array}{c}\text { Promedio municipal por } \\
\text { Departamento (\%) }\end{array}$ \\
\hline Atlántico & 74,75 \\
Bolívar & 58,76 \\
Córdoba & 64,88 \\
Cesar & 61,07 \\
La Guajira & 64,62 \\
Magdalena & 58,71 \\
Sucre & 60,14 \\
San Andrés & 51,74 \\
\hline
\end{tabular}

Fuente: elaborado con información del Departamento Nacional de Planeación (Departamento Nacional de Planeación Grupo de Estudios Territoriales, 2016) 
Ahora bien, aunque está legislado que los municipios deben incluir información sobre su gestión para obtener disponibilidad presupuestal para las obras, existe un porcentaje significativo de municipios en la zona Caribe que no cumplen este requerimiento (Tabla 3). Según el Departamento Nacional de Planeación (2016), particularmente, para la región Caribe tan solo el 9\% de los municipios fueron categorizados con desempeño sobresaliente, siendo los municipios del Atlántico los de mejores calificaciones. Como se mencionó en la Tabla 2, este Departamento es el único de la región Caribe que tiene Sobresaliente en lo que a gestión municipal se refiere (Departamento Nacional de Planeación Grupo de Estudios Territoriales, 2016). Surge entonces la hipótesis, aún por comprobar, si esta es la razón por la cual el desarrollo es tan desigual en las regiones.

Tabla 3

Información sobre la gestión según porcentaje de participación de los municipios

\begin{tabular}{cccc}
\hline Departamentos & Total de municipios & $\begin{array}{c}\text { Municipios con información } \\
\text { completa }\end{array}$ & $\begin{array}{c}\text { \% de participación de los } \\
\text { municipios al 2015 }\end{array}$ \\
\hline Atlántico & 23 & 14 & 61 \\
Bolívar & 46 & 18 & 39 \\
Córdoba & 30 & 17 & 57 \\
Cesar & 25 & 15 & 60 \\
La Guajira & 15 & 8 & 53 \\
Magdalena & 30 & 14 & 47 \\
Sucre & 26 & 12 & 46 \\
San Andrés & 1 & 0 & 0 \\
\hline
\end{tabular}

Fuente: elaborado con información del Departamento Nacional de Planeación (Departamento Nacional de Planeación Grupo de Estudios Territoriales, 2016)

Por otro lado, además de la calificación en función de los trámites de gestión, existe el indicador de medición de desempeño fiscal IDF con el cual es posible conocer las principales magnitudes financieras y los indicadores que miden la gestión fiscal de los municipios. Al igual que el IDIM, se definen las siguientes categorías en función del (IDF): solvente (IDF $\geq 80 \%$ ), sostenible (IDF $\geq 70$ y menor a $80 \%$ ), vulnerable (IDF $\geq 60$ y menor a $70 \%$ ), riesgo (IDF $\geq 40$ y menor a $60 \%$ ) y crítico (IDF menor del $40 \%$ ). Estos indicadores están visibles y disponibles con el fin de dar cumplimiento al artículo 79 de la Ley 617 del 2000 que reza que debe exhibirse el análisis del comportamiento fiscal de los municipios y departamentos para que la ciudadanía se informe y contraste los resultados con la realidad y la gestión de los mandatarios:

\footnotetext{
“Artículo 79. Control social a la gestión pública territorial. El departamento Nacional de planeación publicará en medios de amplia circulación nacional con la periodicidad que señale el reglamento y por lo menos una vez al año, los resultados de la evaluación de la gestión de todas las entidades territoriales, incluidos sus organismos de control, según la metodología que se establezca para tal efecto” (Ley 617 del 2000, 2000).
}

Además, estos reportes son de utilidad para los entes estatales responsables de la auditoria de la gestión sectorial y su relación con las finanzas (Departamento Nacional de Planeación-Subdirección de Finanzas Públicas Territoriales, 2016). En el Informe del Departamento Nacional de Planeación (2016) se menciona que la región Caribe tiene un IDF de 67.6\% lo cual la ubica como una región vulnerable, que no tiene posibilidad de generar excedentes, pero aun así aporta al 15\% del producto interno bruto colombiano (PIB), superando tan solo en un 2\% a la región Pacífica (Dinero.com, 2015).

Con relación al PIB, específicamente, los Departamentos de Atlántico y Bolívar, cada uno, aporta $4.1 \%$ del PIB frente al 0.2\% de San Andrés y Providencia (Departamento Administrativo Nacional 
de Estadística DANE, 2016). A pesar de las condiciones con las problemáticas pendientes de resolver, el departamento del Atlántico estuvo dentro de los departamentos con mayor crecimiento económico durante el 2015, esto motivado por el crecimiento del sector de la construcción, la industria manufacturera y los establecimientos financieros, seguros, actividades inmobiliarias y servicios a las empresas (Departamento Administrativo Nacional de Estadística DANE, 2016). Por su parte, La Guajira presentó crecimiento en comercio, reparación, restaurantes y hoteles, principalmente. Los otros departamentos aportaron en menor proporción al PIB, con valores bajos de participación en Córdoba (1.7\%), Cesar $(1.8 \%)$, Magdalena (1.3\%), La Guajira (1\%), Sucre (0.8\%). Probablemente, con mejores condiciones de infraestructura, la región podría aportar más al país.

\section{Conclusiones}

El desarrollo económico y social de una comunidad se ve afectado por la ausencia o por la falta de adecuada gestión de parte de los entes gubernamentales. Aunque en los Planes de Ordenamiento Territorial se realiza un diagnóstico de necesidades, en algunas comunidades la disponibilidad presupuestal para los proyectos responde a criterios de priorización que pueden resultar no claros, por lo cual están generando una brecha entre lo planeado y lo ejecutado. Resulta preocupante que no todos los municipios estén diligenciando o realizando trámites mediante los sistemas de información que el Gobierno Nacional de Colombia ha establecido para este fin, pudiendo ser este uno de los aspectos de forma, es decir, subsanables, por las cuales muchas necesidades identificadas no son tratadas oportunamente o se atienden, pero no se reportan y el desconocimiento de su ejecución no permite visibilizar el verdadero desarrollo de los municipios. Por otro lado, el estudio reveló que los ciudadanos cuentan con herramientas como el SECOP, los documentos de rendición de cuentas de los municipios y los boletines que definen los indicadores de desempeño integral municipal y el desempeño fiscal municipal, de modo que todos estamos en condiciones de realizar la veeduría correspondiente y exigirle al gobierno central aclaraciones respecto a las inversiones que se realizan.

\section{Referencias}

Alcaldía de Montería. (2009). Proceso de Revisión y ajuste al POT de Montería. Montería, Colombia. Recuperado el 5 de Febrero de 2017, de http://cdim.esap.edu.co/BancoMedios/Documentos\%20 PDF/monteriacordobapot2002-2015.pdf

Alcaldía de Montería. (2015). Montería. Montería, Colombia. Obtenido de http://www.monteria.gov.co/ Alcaldía de Riohacha. (2016). Nuestro municipio. La Guajira, Colombia. Obtenido de http://www. riohacha-laguajira.gov.co/informacion_general.shtml

Alcaldía de Santa Marta. (2016). Nuesto municipio. http://www.santamarta.gov.co/portal/index.php. Obtenido de http://www.santamarta.gov.co/portal/index.php

Alcaldía de Sincelejo. (2016). Nuestro municipio. Colombia: http://sincelejo-sucre.gov.co/index.shtml. Obtenido de http://sincelejo-sucre.gov.co/index.shtml

Alcaldía de Valledupar. (2016). Nuestro municipio. http://www.valledupar-cesar.gov.co/index.shtml\#5. Obtenido de http://www.santamarta.gov.co/portal/index.php

Alcaldía Distrital de Cartagena de Indias. (2016). Nuestro municipio. Obtenido de http://www.cartagena. gov.co/

Banco de la República, Gersón Javier Pérez y otros. (2014). Economía de las grandes ciudades en Colombia: Seis estudios de caso (Primera ed.). (L. A. Galvis, Ed.) Bogotá: Nomos S.A.

Colombia compra eficiente. (29 de Marzo de 2017). Obtenido de https://www.colombiacompra.gov.co/ compradores/secop-i

Departamento Administrativo Nacional de Estadística DANE. (14 de Octubre de 2016). Cuentas Departamentales - Colombia Producto Interno Bruto PIB. (D. d. Hoyos, Ed.) Bogotá, Cundinamarca, 
Colombia. Recuperado el 31 de Marzo de 2017, de https://www.dane.gov.co/files/investigaciones/ pib/departamentales/B_2005/Bol_dptal_2015preliminar.pdf

Departamento Nacional de Planeación Grupo de Estudios Territoriales. (2016). Evaluación del Desempeño Integral de los Municipios y Distritos, Vigencia 2015. Informe Nacional de Resultados Leyes 152 de 1994, 617 de 2000 y 715 de 2001, Bogotá. Recuperado el 31 de Marzo de 2017, de https://colaboracion.dnp.gov.co/CDT/_layouts/15/WopiFrame.aspx?sourcedoc=\%2FCD T\%2FDesarrollo\%20Territorial\%2FEvaluaci\%C3\%B3n\%20Desempe\%C3\%B1o\%20Integral\%20 2015\%2Epdf\&action=view

Departamento Nacional de Planeación-Subdirección de Finanzas Públicas Territoriales. (2016). Desempeño fiscal de los departamentos y municipios, 2015. Bogotá: Imprenta Nacional de Colombia. Recuperado el 31 de Marzo de 2017.

Departamento para la Prosperidad Social, Programa de las Naciones Unidas para el Desarrollo, Ministerio de Trabajo, Univesidad de la Guajira. (2013). Análisis de la demanda laboral y perfiles ocupacionales en el municipio de Riohacha, departamento de La Guajira. Editorial Universitaria Universidad de la Guajira.

Dinero.com. (22 de Octubre de 2015). Datos curiosos de las regiones y de los departamentos de Colombia. Recuperado el 22 de Marzo de 2017, de http://www.dinero.com/economia/articulo/ pib-departamentos-colombia-2014/215020

Gobernación de Atlántico. (2016). Presentación. Atlántico: http://www.atlantico.gov.co/index.php/ departamento/presentaciondepartamento-45237

Ley 617 del 2000. (9 de Octubre de 2000). Diario oficial № 44.188. Bogotá, Colombia. Obtenido de http://www.auditoria.gov.co/Biblioteca\%20General/Leyes/Leyes_1990-2009/Ley_617_ de_2000_(Racionalizacion_del_Gasto_Publico).pdf

Ministerio de Agricultura y Desarrollo Rural República de Colombia. (2013). Alianza para el fortalecimiento de la producción y comercialización de miel de abejas con productores de la asociación de apicultores de Sucre-APISUCRE en el municipio de Sincelejo Departamento de Sucre. Sincelejo.

Ministerio de Ambiente, Vivienda y Desarrollo Territorial. (Julio de 2004). Serie Planes de Ordenamiento Territorial. Guía Metodológica. Bogotá, Colombia. Recuperado el 29 de Marzo de 2017, de http://www.minvivienda.gov.co/POTPresentacionesGuias/Gu\%C3\%ADa\%20 Formulaci\%C3\%B3n\%20Planes\%20Ordenamiento.pdf

Observatorio del Caribe Colombiano. (2015). Región Caribe Colombiana. Cartagena de Indias, Colombia. Recuperado el 25 de Enero de 2017, de http://www.ocaribe.org/region-caribe

SECOP LP-DT-ATL-001-2014.(14 de Septiembre de 2016). Instituto Nacional de Vías. Magdalena, Colombia: https://www.contratos.gov.co/consultas/detalleProceso.do?numConstancia=14-1-128133.

SECOP Proceso L.P. 042-2015. (25 de Agosto de 2015). Licitación Pública. Estudio, diseño y construcción de pavimento. Montería, Colombia: https://www.contratos.gov.co/consultas/detalleProceso. do? numConstancia=15-1-143236

SECOP Proceso LIC-SI-012-2014. (22 de Julio de 2015). Bolívar-Gobernación. Bolívar, Colombia: https://www.contratos.gov.co/consultas/detalleProceso.do?numConstancia=14-1-116894

SECOP Proceso LP-003-OP-2013. (29 de Diciembre de 2015). Sucre-Alcaldía Municipio de Sincelejo. Sucre, Colombia: https://www.contratos.gov.co/consultas/detalleProceso. do? numConstancia=13-1-90840

SECOP Proceso LP-004-OP-2014. (11 de Diciembre de 2015). Sucre. Obtenido de https://www.contratos.gov.co/consultas/detalleProceso.do?numConstancia=14-1-113051

SECOP Proceso LP-005-OP-2014. (18 de Diciembre de 2015). Sucre Alcaldía Sincelejo. sucre, Colombia: https://www.contratos.gov.co/consultas/resultadoListadoProcesos.jsp\#

SECOP Proceso LP-006-2013. (26 de Octubre de 2014). Sucre-Gobernación. Sucre, Colombia: https:// www.contratos.gov.co/consultas/detalleProceso.do?numConstancia=13-1-90842 
SECOP Proceso LP-010-2014. (11 de Diciembre de 2015). Sucre-Alcaldia Municipio de Sincelejo. sucre: https://www.contratos.gov.co/consultas/detalleProceso.do?numConstancia=14-1-118279

SECOP Proceso LP-012-2014. (9 de Octubre de 2015). Sucre Gobernación. Sincelejo, Colombia: https:// www.contratos.gov.co/consultas/detalleProceso.do?numConstancia=14-1-124215

SECOP Proceso LP0132013. (17 de Diciembre de 2014). La Guajira- Alcaldía Municipio de Riohacha. La guajira, Colombia: https://www.contratos.gov.co/consultas/detalleProceso. do?numConstancia=13-1-106114

SECOP Proceso LP-DO-SRN-015-2014. (29 de Octubre de 2015). Instituto Nacional de Vías. Valledupar, Colombia: https://www.contratos.gov.co/consultas/detalleProceso. do?numConstancia=14-1-120885

SECOPProcesoLP-DO-SRN-015-2014.(08 de Juliode2016).InstitutoNacionalde Vías. Valledupar,Colombia: https://www.contratos.gov.co/consultas/detalleProceso.do?numConstancia=14-1-120885

SECOP Proceso LP-DO-SRN-024-2014. (21 de Septiembre de 2016). Instituto Nacional de Vías. Atlántico, Colombia: https://www.contratos.gov.co/consultas/detalleProceso. do?numConstancia=14-1-121758

SECOP Proceso LP-DO-SRN-026-2014. (5 de Diciembre de 2016). Instituto Nacional de Vías. Magdalena, Colombia: https://www.contratos.gov.co/consultas/detalleProceso. do?numConstancia=14-1-121765

SECOP Proceso LP-DT-ATL-001-2014. (14 de Septiembre de 2016). Instituto Nacional de Vías. Bolívar, Colombia: https://www.contratos.gov.co/consultas/detalleProceso. do?numConstancia=14-1-128133

SECOP Proceso LP-DT-ATL-001-2014. (14 de Septiembre de 2016). Instituto Nacional de Vías. Bolívar, Magdalena, Atlántico, Colombia: https://www.contratos.gov.co/consultas/detalleProceso. do?numConstancia=14-1-128133

SECOP Proceso LP-DT-MAG-001-2014. (6 de Septiembre de 2016). Instituto Nacional de Vías. Magdalena, Colombia: https://www.contratos.gov.co/consultas/detalleProceso. do?numConstancia=14-1-128163

SECOP Proceso LP-SGT-OPA-052-2013. (09 de Septiembre de 2014). Instituto Nacional de Vías. Sucre, Colombia: https://www.contratos.gov.co/consultas/detalleProceso. do?numConstancia=13-1-103239

SECOP Proceso UNIBAC-LIC-002-2016. (15 de Noviembre de 2016). Bolívar- Institución Universitaria Bellas Artes y Ciencias de Bolívar. Bolívar, Colombia: https://www.contratos.gov.co/consultas/ detalleProceso.do?numConstancia=16-1-157724

wikimedia con información de IGAC. (2012). wikimedia.org. Obtenido de De Shadowxfox - Trabajo propio http://geoportal.igac.gov.co/mapas_de_colombia/IGAC/Tematicos2012/RegionesGeograficas.pdf, CC BY-SA 4.0, https://commons.wikimedia.org/w/index.php?curid=36272267 